\title{
Crowdworking und Leistungsgerechtigkeit
}

\section{Ansprüche von Crowdarbeitenden an distributive, prozedurale und informationale Gerechtigkeit}

\author{
Sabine Pfeiffer $(\mathbb{D}) \cdot$ Sandra Kawalec $\cdot$ Max Held $\cdot$ Verena Held
}

Eingegangen: 10. Mai 2019 / Angenommen: 20. Juni 2019 / Online publiziert: 28. Juni 2019

(C) Der/die Autor(en) 2019

Zusammenfassung Über Plattformen vermittelte Crowdwork ist eine noch junge Arbeitsform. Sie ist formell nicht an ein Arbeitsverhältnis oder eine Betriebsorganisation gebunden. Der Beitrag geht der Frage nach, ob sich mit dem Auflösen dieser institutionellen Bindungen auch traditionell geprägte Ansprüche an Arbeit verschieben. Typisch für Crowdwork ist einerseits eine geringere Vergütung, ein volatiles Einkommen und fehlende Sozialabgaben, andererseits fehlen die Vergleichs- und Aushandlungsmöglichkeiten einer Betriebsorganisation. Daher stellt sich die Frage, ob sich Ansprüche an die Leistungsgerechtigkeit mit dieser Arbeitsform verändern. Dazu untersuchen wir die subjektiven Ansprüche von Crowdarbeitenden auf unterschiedlichen Typen von Crowdworking-Plattformen im deutschsprachigen Raum. Die explorativ angelegte Forschung zeigt: Crowdarbeitende bewerten das wettbewerbsorientierte Plattformsystem mit ähnlichen Leistungsgerechtigkeitsansprüchen

Die empiriischen Arbeiten für diesen Artikel entstanden im Rahmen eines Stipendiums der Hans-Böckler-Stiftung, die konzeptionellen Arbeiten im Vorhaben „Gute agile Projektarbeit in der digitalisierten Welt (diGAP)“, gefördert vom Bundesministeriums für Bildung und Forschung (BMBF) und dem Europäischen Sozialfonds (ESF) im Rahmen der Förderprogramme „Zukunft der Arbeit“ und „Innovationen für die Produktion, Dienstleistung und Arbeit von morgen“" und betreut vom Projektträger Karlsruhe (PTKA).

S. Pfeiffer $(\bowtie) \cdot$ M. Held $\cdot$ V. Held

Lehrstuhl für Soziologie (Technik - Arbeit - Gesellschaft), Friedrich-Alexander-Universität

Erlangen-Nürnberg, Fürther Str. 246c, 90429 Nürnberg, Deutschland

E-Mail: sabine.pfeiffer@fau.de

M. Held

E-Mail: maximilian.held@fau.de

V. Held

E-Mail: verena.vk.held@fau.de

S. Kawalec

Lehrstuhl für Soziologie, Universität Hohenheim, Wollgrasweg 23, 70599 Stuttgart, Deutschland

E-Mail: sandra.kawalec@uni-hohenheim.de 
traditioneller Beschäftigung und sie sind aus dieser Perspektive mit der distributiven und prozeduralen Leistungsgerechtigkeit des Crowdworking eher unzufrieden.

Schlüsselwörter Klickarbeit · Plattformökonomie · Gig-Ökonomie · Mikrojobs · Solo-Selbständige

\section{Crowdworking and Perceived Fairness}

Crowdworkers' Expectations Concerning Distributive, Procedural, and Informational Justice

Abstract Crowdwork, or distributed and platform-mediated contract work, is a relatively new form of work potentially challenging existing fairness norms. Crowdwork is often characterised by low and volatile incomes without benefits, but traditional labour market institutions to negotiate industrial relations are inoperative for such contract work.

Though formally self-employed, crowd workers expectations of fairness may be deeply shaped by and expressed through traditional labor market institutions such as labor law, unions and compensation, none of which apply to nascent crowd work.

We study the subjective expectations of workers on three German-language crowdworking platforms specialized on testing, design and micro-jobs. Using an exploratory design, we find that crowd workers judge their pay and assessment by standards borrowed from traditional employment. Many are dissatisfied with the procedures and outcomes of their performance-based compensation.

Keywords Clickwork $\cdot$ Platform economy $\cdot$ Gig economy $\cdot$ Microjobs $\cdot$ Selfemployed

\section{Alles neu ...? Zur Einleitung}

Seit im Jahr 2011 der Begriff „Industrie 4.0“ im Rahmen der Hannover Messe der Öffentlichkeit vorgestellt wurde (Kagermann et al. 2011), ist in Deutschland der Diskurs zur digitalen Transformation von Arbeit mit der ,4.0“ eng verknüpft. Während „Industrie 4.0“ sich enger auf produzierende Geschäftsmodelle und vier Technologiesprünge (Dampfmaschine, Elektrifizierung, Informatisierung und nun cyber-physische Systeme) bezieht, zielt das Bundesministerium für Arbeit und Soziales mit dem Begriff „Arbeit 4.0“ (BMAS 2015, S. 34-35) auf den Wandel der Formen und Institutionen des Erwerbsverhältnisses: Von den Anfängen der Industriegesellschaft mit ersten Organisationen von Arbeitern/-innen Ende des 18. Jahrhunderts (1.0), den Beginn der Massenproduktion mit den Anfängen des Wohlfahrtsstaats (2.0) bis zur Globalisierung und der sozialen Marktwirtschaft (3.0) und schließlich vernetztes Arbeiten, Wertewandel und sozialer Kompromiss (4.0). Die Phasen 1.0 bis 3.0 waren geprägt von der Institution des Erwerbsverhältnisses: Bezahlte Arbeit findet auch heute noch vor allem im Rahmen abhängiger Beschäftigung statt und das bedeutet für die Mehrheit der Beschäftigten immer noch überwiegend unbefristete Arbeitsverträge und meist eine Tätigkeit innerhalb einer physischen Betriebsorganisation. 
Alle sozialen Absicherungen (Unfall, Arbeitslosigkeit, Alter) und damit letztlich die Institution des Wohlfahrtsstaats als Ganzes hängen weitgehend von diesem Modell der Erwerbsarbeit ab.

Crowdworking aber bricht mit diesem Modell. Verstanden wird darunter eine bezahlte Form des Crowdsourcing und damit ein ,neues Prinzip der Arbeitsorganisation: Arbeit wird nicht mehr kraft Direktionsrecht individuell zugewiesen, sondern die Bearbeitenden wählen ihre Arbeit selbst“" (Mrass und Leimeister 2018, S. 139). Im Gegensatz zum Outsourcing, bei welchem bisher intern erbrachte Leistungen in größeren Auftragsbündeln und in Form fester Leistungsverträge an andere Unternehmen verlagert werden, werden beim Crowdsourcing einzelne - und oft kleinteilige - Aufträge punktuell an eine Masse von unbekannten Akteuren über einen offenen Aufruf auf einer Internetplattform - dem Intermediär - angeboten. Zwischen Arbeitskraftanbieter und -nutzer besteht kein direktes und längerfristiges Vertragsverhältnis - im Gegenteil: das höchst flexible und punktuelle sowie gleichzeitig über eine Plattform in wettbewerblich vermittelte Aufeinandertreffen beider Seiten wird deshalb als ein ,new template of work“ bezeichnet (Bollier 2011, S. 14). Noch ist die Zahl der Crowdarbeitenden in Deutschland gering (Huws et al. 2017; Pongratz und Bormann 2017). Würde Crowdwork allerdings zu einem vorherrschenden Modell und damit Solo-Selbständigkeit in großer Zahl bisherige Erwerbsformen verdrängen, hätte das immense Folgen für alle Institutionensysteme des Arbeitsmarkts und des Wohlfahrtsstaats. Auch wenn aktuell die Frage nach dem Ende der vertraglichen Erwerbsarbeit noch nicht auf der Agenda steht, scheint Regelungsbedarf zu bestehen: Nicht nur in der internationalen Forschung (Berg 2016; Graham et al. 2017; Mandl und Curtarelli 2017), sondern auch in Deutschland wird die Notwendigkeit einer Regulierung - insbesondere im Hinblick auf Arbeitsrecht, Datenschutz, Mitbestimmung, Einkommenssicherheit und -höhe sowie Absicherung - rund um Crowdwork bereits virulent und konkret diskutiert (vgl. Benner 2014; Dabrowski und Wolf 2017).

Eine von acht in Deutschland angesiedelten Crowd-Plattformen hat 2017 einen „Code of Coduct“ unterzeichnet, der sich als „Leitfaden für eine gewinnbringende und faire Zusammenarbeit zwischen Crowdsourcing-Unternehmen und Crowdworkern“ versteht (Deutschen Crowdsourcing Verband 2017). Ein ganz wesentlicher Punkt ist dabei ,faire Bezahlung“. Der Code of Conduct aber lässt offen, was Crowdarbeitende in Deutschland als fair bzw. gerecht empfinden. Dafür müsste zunächst geklärt werden, ob sich die Ansprüche an Leistungsgerechtigkeit an Crowdarbeit anders darstellen als an klassische und regulierte Erwerbsarbeit. Offen ist zunächst auch, ob sich die Ansprüche verändern, wenn der Vergleich mit der Leistung anderer nicht mehr möglich ist, weil der betriebliche Kontext fehlt. Diesen Fragen nach Ansprüchen an die Leistungsgerechtigkeit im Crowdworking geht dieser Beitrag nach. Dazu wird zunächst der Forschungsstand zu Crowdwork und zu Leistungsgerechtigkeit referiert und im zweiten Schritt werden empirische Ergebnisse aus qualitativen und quantitativen Erhebungen mit Crowdarbeitenden von verschiedenen Plattformtypen in Deutschland vorgestellt. 


\section{Crowdwork: Leistung jenseits von Betrieb und Arbeitsvertrag}

Crowdwork kann allgemein definiert werden als aktuellstes Phänomen ,,[...] der Strategie des Auslagerns einer üblicherweise von Erwerbstätigen entgeltlich erbrachten Leistung durch eine Organisation oder Privatperson mittels eines offenen Aufrufes an eine Masse von unbekannten Akteuren“ (Papsdorf 2009, S. 69). Crowdwork weist „nur partielle Überschneidungen“ mit der Sharing Economy auf (Hertwig und Papsdorf 2017, S. 526). In der Literatur finden sich vielfältige Versuche einer Typologisierung: So unterscheiden Mrass und Leimeister Crowdwork entlang von sieben Plattformtypen: Context-/Texterstellung, Design, Innovation, Marktplatz, Mikrotask, Testing und Kundenberatung/Marktforschung/Vertrieb (2018, S. 142). Andere beziehen neben der Plattformart weitere Dimensionen ein etwa den Arbeitskraftpool, die Vertragsarten, die Formen der algorithmischen Kontrolle oder Quellen und Mechanismen für das Entstehen eines ,digital trust“ (Howcroft und Bergvall-Kåreborn 2019). Die beiden Autorinnen unterscheiden im Kern vier Plattformarten, dabei ist ein Kriterium, ob die Tätigkeit verlässlich bezahlt wird oder nicht (paid vs. nonpaid or speculative) und ein zweites, wer der initiierende Akteur ist: die Arbeitenden (z.B. spezialisierte Freelancer) oder die Auftraggeber. Eine Unterscheidung auf Basis von qualitativen Experteninterviews differenziert für den deutschsprachigen Raum zwischen den vier zentralen Typen Innovations-, Testing-, Microjob- und Designplattformen (Kawalec 2019).

Die Schwierigkeit einer klaren Definition erschwert Einschätzungen zur Verbreitung, erst recht, will man das globale Phänomen für Deutschland quantifizieren. Anders als in den USA wird die wirtschaftliche Bedeutung von Crowdworking-Plattformen in Deutschland noch als gering eingeschätzt: Anfang 2017 haben 32 Crowdworking-Plattformen ihren Sitz oder zumindest einen (physischen) Standort in Deutschland (Mrass und Leimeister 2018, S. 142). Im weltweiten Vergleich sei in Deutschland ,sowohl Nachfrage als auch Angebot relativ niedrig“ (Pongratz und Bormann 2017, S. 165).

Plattformen nennen keine belastbaren Zahlen zu ihren aktiven Crowdarbeitenden, sie behandeln diese entweder als Geschäftsgeheimnis oder werben mit - wahrscheinlich überzogenen - Zahlen. Zudem ist davon auszugehen, dass Crowdarbeitende auf mehr als einer Plattform aktiv sind, damit wäre der Versuch einer Quantifizierung über Plattformangaben auch bei mehr Zahlentransparenz generell von begrenztem Nutzen. Zwei Studien versuchen sich auf nachvollziehbarer und - soweit eben möglich - seriöser Basis an einer Abschätzung zur zahlenmäßigen Relevanz von Crowdarbeitenden in Deutschland:

- Eine Vergleichsstudie (Huws et al. 2017) schätzt auf der Basis einer repräsentativen Online-Erhebung $(N=2180)$, dass 1,45 Mio. Crowdarbeitende und damit 2,5\% der deutschen Erwerbsbevölkerung mindestens 50\% ihres Einkommens aus dieser Arbeitsform beziehen. In einer engeren Definition fasst die Studie eine „Kerngruppe“, d.h. Personen, die sich zudem über eine spezielle App über angebotene Arbeiten informieren; diese ,,professionellen“ Crowdarbeitenden schätzt die Studie für Deutschland auf 1,07 Mio. oder 1,9\% der Erwerbsbevölkerung. 
- Pongratz und Bormann sind in ihrer Einschätzung vorsichtiger und kritisieren die Studie von Huws et al. aus forschungsmethodischen Gründen als ,irreführend“ (2017, S. 180). Sie gehen in Deutschland von 500.000 bis zu einer Million Personen aus, die in den vergangenen zehn Jahren ein noch bestehendes Profil auf einer Plattform für Online-Arbeit angelegt haben; von diesen nehmen 100.000 bis 300.000 mindestens einmal monatlich einen Auftrag an, nur bei 1000 bis 5000 Personen sei dabei jedoch von einer erwerbssichernden Einnahme (im Vergleich zum deutschen Durchschnittslohn) auszugehen (Pongratz und Bormann 2017, S. 167).

Crowdwork kann für die Arbeitenden durchaus Vorteile bringen, insbesondere in Regionen mit schwach entwickelten Arbeitsmärkten, insofern konstatiert eine Langzeitstudie zu Crowdwork in Sub-Sahara und Südostasien: „There is no simple story of exploitation (...)“ (Graham et al. 2017, S. 153). Allerdings zeigt die Studie auch, dass die Risiken dieses Arbeitsmodells für die Arbeitenden steigen, je mehr sie damit ihren Lebensunterhalt bestreiten. Auch eine Studie auf Plattformbetreibern aus Deutschland (Mrass und Leimeister 2018, S. 145-148) zeigt, dass Crowdarbeitende von mehr Flexibilität und dem Zuverdienst profitieren können, dass sie aber mit relativ geringer Bezahlung ohne Sozialversicherung und Einkommensschwankungen konfrontiert sind; dagegen profitierten die Crowdsourcer nicht nur von der Flexibilität und Nutzung externen Know-Hows, sondern insbesondere von den geringen Kosten und eingesparten Sozialabgaben.

Die neuen digitalen Arbeitsformen scheinen damit „alte“ Interessengegensätze wieder aufzurufen, die mit Einkommenshöhe und -sicherheit zu tun haben und in herkömmlicher Beschäftigung üblicherweise über unterschiedliche Institutionen des Arbeitsmarkts und betriebliche Aushandlungsprozesse hergestellt werden. Das ist bei Crowdwork kein zufälliges Regelungsresiduum, sondern systematisch angelegt. Denn: Im Vergleich zu anderen Formen plattformvermittelter Arbeit, deren eigentliche Durchführung in der realen Welt stattfindet, zeichnet sich die rein Online stattfindende Crowdwork durch die zwei Charakteristika der „Deterritorialisierung “ und „Entpersonalisierung “ von Arbeit aus (Menz und Tomazic 2017, S. 14-15; Hervorh. i. O.). Berührt sind damit einerseits die distributive Leistungsgerechtigkeit, da damit das globale Lohngefälle und lokale Lebenshaltungskosten entkoppelt werden; und andererseits die prozedurale Leistungsgerechtigkeit, da es an Transparenz und Einflussnahme bei der Bewertung und deren Kriterien mangelt und der Betrieb als sozialer Kontext fehlt - was für die Crowdarbeitenden nicht nur emotionale Folgen hat (vgl. Petriglier et al. 2018).

\section{Leistungsgerechtigkeit und Arbeit}

Das Thema Gerechtigkeit am Arbeitsplatz hat mit der qualitativen Studie von Dubet (2008) neue wissenschaftliche Aufmerksamkeit erfahren. Auf der Basis von 350 Interviews werden drei Basisprinzipien herausgearbeitet: Gleichheit, Leistung und Autonomie. Dubet konzeptualisiert seine Gerechtigkeitsprinzipien als „,normative Aktivität“ im doppelten Sinne: zur „Gesamtheit von Prinzipien, die als legitim gelten“ gesellt sich die ,autonome[ ] Entscheidung, eines selbstständigen Urteils“ 
(Dubet 2008, S. 17). Leistungsgerechtigkeit umfasst dabei, was als ein gerechter Tausch zwischen Leistung und Gegenleistung empfunden wird (Dubet 2008, S. 24). Für Dubet erfasst dabei der Leistungsbegriff sowohl „das objektive Ergebnis der Handlung“ wie den „Einsatz des Akteurs dieser Handlung“, die „Ambivalenz der Leistung“ bewege sich damit immer ,zwischen objektivem Nutzen und erbrachter Anstrengung“ (2008, S. 127). Anknüpfend an Dubet sind in den letzten Jahren zwei umfangreiche qualitative Studien zu Gerechtigkeitsansprüchen von Beschäftigten in Deutschland entstanden.

- Hürtgen und Voswinkel (2014) führten 42 biografische Interviews, bei der das Sample bewusst rekrutiert wurde aus der „Mitte“ der Beschäftigten, d. h. Erwerbspersonen mit unbefristeten Arbeitsverträgen, mit mittlerer Qualifikation und von mittlerem Lebensalter (2014, S. 39-40). Demnach entwickelten Beschäftigte aus grundlegenden Auffassungen von sich als Leistungskraft, als menschlichem Wesen und als Sozialwesen „Werte von allgemeiner normativer Geltung“. Ansprüche an das Einkommen werden aus einer Perspektive der Leistungskraft verstanden als adäquater Verdienst für den eigenen Arbeitseinsatz (Hürtgen und Voswinkel 2014, S. 139-140); aus einer Perspektive der sozialen Existenz ist der Gradmesser für ein gutes Einkommen zudem verbunden mit dessen „Stimmigkeit“ in Bezug auf die eigene Lebensgestaltung, mit Ansprüchen an die Planungssicherheit, an als verdient empfundene Sozialleistungen und Rente (Hürtgen und Voswinkel 2014, S. 145-150). Insgesamt werde das erzielte Einkommen sowohl ,als Gegenwert zu einer [...] erbrachten Leistung“ verstanden wie als ,,symbolische Anerkennung der eigenen Leistungskraft“" (Hürtgen und Voswinkel 2014, S. 149).

- Eine weitere arbeitssoziologische Studie (Kratzer et al. 2015; ähnlich auch Tullius und Wolf 2016) geht den Legitimationsmustern von Arbeit auf der Basis von 320 qualitativen Interviews mit Beschäftigten ohne Leitungsfunktion in Deutschland nach. Neben Ansprüchen an die Selbstverwirklichung am Arbeitsplatz, an die Beteiligung bei Entscheidungen im unmittelbaren Arbeitsumfeld sowie an die Wahrung der Würde zählen dazu auch subjektive Ansprüche an die Leistungsgerechtigkeit als einem aufwandsbezogenen Begriff. Als Legitimationsanspruch fassen die Autoren/-innen normative Erwartungen von Beschäftigten, die ,explizit oder implizit hinsichtlich bestimmter betrieblicher Ordnungen (z. B. Verteilungsregeln, Entscheidungsprozeduren, Maßnahmen der Krisenbewältigung) formuliert werden“ (Kratzer et al. 2015, S. 14). Dabei werde der Maßstab für Leistungsgerechtigkeit auf Facharbeitsebene mit der Leistungsverausgabung im Arbeitsprozess und in der sog. Wissensarbeit mit der Leistungsbereitschaft verknüpft (2015, S. 50-51).

Neben diesen qualitativen Studien, welche die Komplexität verschiedener Anspruchsdimensionen an Arbeit verstehbar machen, zeigt eine quantitative Studie (Schneider 2018) auf der Basis des Sozioökonomischen Panels (SOEP), dass sich die Mehrheit der abhängig Beschäftigten in Deutschland als gerecht entlohnt empfindet: Knapp $61 \%$ (64\% mit Tarifbindung) empfinden ihren Bruttoverdienst, jedoch nur 55\% (56\% mit Tarifbindung) der Befragten ihren Nettoverdienst als gerecht. Der Bruttoverdienst wird über fast alle Gruppen hinweg als gerechter empfunden als der Nettoverdienst. Auffällig ist, dass Vollzeitbeschäftigte mit 59\% ihren Bruttover- 
dienst seltener als gerecht betrachten als Teilzeitbeschäftigte $(62 \%)$ und der Wert bei geringfügig Beschäftigten mit $79 \%$ besonders hoch ist (2018, S. 366).

Selbst bei Minijobs, die wie Crowdwork oft als Zuverdienst und im Niedriglohnbereich angesiedelt sind, die nicht grudsätzlich oder nur gering zur sozialen Absicherung beitragen und zudem oft (etwa im Einzelhandel) mit Rufbereitschaft ohne garantierte Mindeststunden organisiert sind, ist $77 \%$ der Beschäftigten ein gutes Einkommen und $82 \%$ ein leistungsgerechtes Einkommen wichtig bis sehr wichtig (Beckmann 2019, S. 251-342). Beide Dimensionen zählt die 2016 erhobene Studie $(N=1004)$ zu den ,Flops“ beim Match von Soll- und Istzustand: letztere decken beim guten Einkommen für $47 \%$ und beim leistungsgerechten Einkommen für $48 \%$ der Befragten (2019, S. 293).

Ergebnisorientierung ist alles andere als exklusiv für Crowdwork, auch innerhalb von Erwerbsverhältnissen hat sich die betriebliche Leistungspolitik in den letzten Jahren immer mehr von einer Aufwands- zu einer Ergebnisorientierung verschoben (vgl. Breisig 2018) und diese Verschiebung der betrieblichen Steuerungsverfahren vom Aufwand hin zu Erfolg oder Ertrag, so konstatieren Menz und Niess, erscheine den Beschäftigten ,nicht grundsätzlich illegitim“, konstituiere aber auch ,kein eigenständiges Gerechtigkeitsprinzip“ (2018, S. 133; siehe Fußnote 12). Exklusiv für Crowdwork ist aber, dass sie sich anders als die zitierten Studien zu Leistungsgerechtigkeit - das gilt auch für Minijobende - nicht im Kontext betrieblicher Arenen abspielt. Gerade dort aber, also im Betrieb, konkretisiere sich ,die Frage nach der Ausformung dieses Prinzips“ der Leistungsgerechtigkeit (Menz und Nies 2018, S. 132-133) und aufgrund von wiederholten Verletzungserfahrungen bleibe der Anspruch auf Leistungsgerechtigkeit ein ,,dauerhafter Konflikt- und Kritikherd in den Betrieben“ (Tullius und Wolf 2016, S. 496-497). Weil bei Crowdwork dieser Kontext und damit der soziale Raum für Konflikt und Kritik fehlt, bleibt damit offen, wie sich die Ausformung des Anspruchs an Leistungsgerechtigkeit vollzieht. Dieser Frage wollen wir nachfolgend weiter nachgehen. Dies erfolgt in zwei explorativen empirischen Schritten: Die zunächst vorgestellten Ergebnisse qualitativer Erhebungen dienen dazu, relevante Dimensionen der Leistungsgerechtigkeit aus Sicht der Crowdarbeitenden besser zu verstehen. Im Anschluss daran wird mit ebenso explorativer Absicht ein Vorschlag zur quantitativen Erfassung vorgestellt und Ergebnisse zu einer entsprechenden Online-Studie diskutiert.

\section{Leistungsgerechtigkeit bei Crowdwork qualitativ verstehen}

Aufgrund der unterschiedlichen Rahmenbedingungen ist fraglich, ob die Ergebnisse des vorgestellten Forschungsstands zu Leistungsgerechtigkeit in klassischer Beschäftigung und im Kontext betrieblicher Organisationen erhoben wurden, auf Crowdwork übertragbar sind. Um Dimensionen der Leistungsgerechtigkeit explorativ und aus der Perspektive der Crowdarbeitenden zu entwickeln war daher zunächst ein eigenständiger qualitativer Schritt nötig. Der dazu verwendete Materialkorpus umfasst insgesamt 36 qualitative Interviews, die sich aus drei Quellen speisen (vgl. ausführlich Kawalec 2019): 
- Zum einen wurden 11 Interviews bei einem großen, auf Crowdsourcing als Teil der Outsourcing-Strategie zeitweise umstellenden IT-Unternehmen in zwei Wellen geführt. Drei Beschäftigte mit Projektleitungsrollen und zwei Betriebsräte sowie ein für das Unternehmen zuständige/r Gewerkschaftsfunktionär/-in ${ }^{1}$ wurden zu Beginn des Projekts und zwei Projektleiter/-innen und zwei Betriebsräte erneut vier Jahre später nach Beendigung des Projekts in 2016 interviewt.

- Zum anderen wurden sieben qualitative Experteninterviews (Gläser und Laudel 2010) mit Expertinnen und Experten aus der Wissenschaft (ein Interview), dem gewerkschaftlichen Kontext (zwei Interviews) und von Plattformbetreibern (vier Interviews aus dem Bereich Testing und Innovation).

- Zum dritten wurden neben diesen 18 Interviews zur vertiefenden Felderschließung weitere 18 Interviews mit Crowdarbeitenden geführt, von denen drei auf Testing-, sieben auf Innovations-, drei auf Design- und weitere fünf auf Microjob-Plattformen tätig waren. Sie differieren nach Geschlecht, Alter (20 bis über 60) und Situation (von Arbeitslosigkeit bis hochqualifizierter Vollzeiterwerbstätigkeit). Sampling und Auswertung folgte der Methode der Grounded Theory (Glaser und Strauss 2009) sowie inhaltsanalytisch; beim Feldzugang wurde teils auf soziale Netzwerke und auf das Schneeballverfahren (vgl. Przyborski und WohlrabSahr 2010) gesetzt. Die Erhebungen wurden zwischen 2016 und 2018 im deutschsprachigen Raum geführt.

Eine zentrale Dimension, bei der die befragten Crowdarbeitenden Gerechtigkeitsansprüche formulieren bzw. deren Verletzung von Seiten der Plattform deutlich benennen, ist die fehlende Planungssicherheit. Da nie klar ist, wie viele Aufträge man erhält und ob man für geleistete Arbeit am Ende auch wirklich Geld bekommt, ist die fehlende Planungssicherheit eine oft thematisierte Gerechtigkeitsdimension, die sich nur abmildert, wenn Crowdwork als Zuverdienst und nicht als grundsätzlich Einkommensquelle dient. Typische Aussagen dazu sind:

Die Nachteile sind halt einfach, dass man halt nicht wirklich fest mit einem Gehalt planen kann, also ich kann jetzt nicht irgendwie sagen, ich bekomme Ende des Monats immer mein festes Gehalt, meine keine Ahnung wie viel Euro, da drauf kann man sich halt nicht verlassen. (Bastian Buchmann, TestingPlattform 11:101)

Du arbeitest an irgendwas. Da steckst du Zeit rein [...]. Und dann wird das halt nicht genommen, dann hast du halt umsonst gearbeitet. [...] - ja du machst halt Arbeit und auch oft viel Arbeit und gute Arbeit und du kriegst dann halt nichts dafür. Und das funktioniert dann halt nicht. Und dann bricht das ganze Kartenhaus so 'n bisschen zusammen (Norman Neuland, Design-Plattform 17:18)

Die Frage der Planungssicherheit ist eng verknüpft mit der Gerechtigkeit der Leistungsbewertung. Hier wird zum einen die Intransparenz der Kriterien beklagt, vor allem aber auch die als meist zu gering empfundenen Möglichkeiten auf Kriterien

\footnotetext{
1 Wegen der mit dem crowdbasierten Outsourcing strategisch und operativ befassten, beschränkten Personenzahl erfolgen die Angaben hier geschlechtsneutral; so soll auch Personen mit Inneneinsicht eine indirekte De-Anonymisierung verwehrt bleiben.
} 
und Prozess der Bewertung Einfluss zu nehmen oder auch Widerspruch geltend zu machen. Letzteres kann auch ganz faktisch scheitern, hier eine exemplarische Schilderung:

Man kennt die auch nicht. Man kann sich auch nicht mit denen auseinandersetzen. Man kann dann denen über den Support Bescheid sagen [...]. Aber für diese kleinen Beträge, die man da kriegt, ja? Also soll ich jetzt für einen Euro Honorar auch noch eine E-Mail an die schreiben [...?]. Dann sage ich mir, „Ach, nein. Da mache ich doch lieber gleich den nächsten Auftrag“ (Melissa Müller, Design-Plattform 22:45).

Problematisch ist auch die Bewertungshistorie, die zu einer Art Aufschichtung von Ungerechtigkeit bei der Leistungsbewertung führen kann.

Es ist dann nur so, wenn man eine bestimmte Anzahl von Ablehnungen hat, kann es sein, dass man an bestimmte Aufträge nicht rankommt. Da steht dann: bloß, wenn man $90 \%$ in der Kategorie oder von dem Auftraggeber richtig hat, dann kann man weitermachen. Und wenn man das nicht hat, wird man für [...] ein paar Wochen, ein paar Monate, wie auch immer, gesperrt und kann dann nichts mehr machen. (Klaus Klein, Microtask-Plattform 21:80)

Empfundene Ungerechtigkeit ist nicht nur ein Phänomen abschließender Bewertung, sondern beginnt schon mit den oft unklaren Aufgabenbeschreibungen. So schildert Gerda Grass, dass die Aufgaben oft „ein bisschen zu unspezifisch“ oder „Wischi-Waschi“" seien (Design-Plattform 16:64). Da an der Aufgabenbeschreibung am Ende wieder die Bezahlung hängt, sind nicht eindeutige oder gar widersprüchliche Aufgabenbeschreibungen nicht nur ärgerlich, sondern berühren tatsächlich das Gerechtigkeitsempfinden, typisch dafür:

Also ich habe denen eben geschrieben, „Wenn ihr so Briefings macht, dann müssen die eben auch eindeutig sein“. Da ging es um Beispielsätze. [...G]anz oben, stand eben, „Du musst mindestens zwei Beispielsätze anführen“, und weiter unten stand, „Ein Beispielsatz ist Pflicht. Zwei wären schön“ (Martin Mönch, Mikrotask-Plattform 24:46)

Unabhängig von der Art der Plattformen empfinden die Befragten die Höhe der Prämien durchgängig als unangemessen. Dabei wird entweder mit anderen Formen von Nebentätigkeiten verglichen „Da habe ich bis jetzt zwei, drei Euro verdient. Das lohnt sich nicht. Die Zeit, die du da anwendest, da gehe ich besser Rasenmähen irgendwo" (Pawel Polanski, Microtask-Plattform 14:22). Andere stellen das geringe zu erzielende Einkommen in Relation zu den Gewinnen der Auftraggeber und Plattformbetreiber: ,[E]in Millionengeschäft eigentlich ist das Ganze. [...] ist schon moderne Sklaverei“ (Fritz Freudig, Innovations-Plattform 15:45). Immer wieder wird auch beklagt, dass geleisteter Aufwand und Bezahlung nicht zusammenpassen, exemplarisch: „Für einen kleinen Lohn wird Großes geleistet, vielleicht für gar keinen Lohn. Sodass das eine Ausbeutung ist." (Sigmund Schlecht, IdeenPlattform 19:41). Den hier nur in Kürze skizzierten Dimensionen Planungssicherheit, Leistungsbewertung, Aufgabenbeschreibung und Prämien nähern wir uns im nächsten Schritt weiterhin explorativ auf quantitativer Basis. 


\section{Leistungsgerechtigkeit bei Crowdwork quantitativ erfassen}

Mittlerweile finden sich erste Studien zur einkommensbezogenen Leistungsgerechtigkeit und Crowdwork (Ye et al. 2017; Alpar und Osterbrink 2018), dabei werden Crowdarbeitende nicht nur nach dem „Perceived Fairness in Pay“ (PFP) befragt, sondern diese Frage wird verbunden mit für diesen Zweck generierten Tests auf Microtask-Plattformen wie MTurk. Dabei zeigt sich ein Zusammenhang von PFP mit der Performance-Qualität (Ye et al. 2017, S. 333). Die Autoren passen drei Items zu PFP für Crowdwork sprachlich leicht an, die verwendeten Formulierungen sind: „(1) ,My payment reflects the effort I have put into the task, '(2) ,My payment is appropriate for the work I have completed, " and (3) ,My payment is justified given my performance “" (2017, S. 330). Basis ist dabei die Skala zur organisationalen Gerechtigkeit (GEO) von Colquitt (2001), die von Maier et al. (2007b) für Deutschland angepasst und auf Validität getestet wurde. Insgesamt umfasst das Instrument vier Skalen zur prozeduralen (7 Items), distributiven (4 Items), interpersonalen (4 Items) und informationalen Gerechtigkeit (5 Items), die Wertebereiche der Likert-Skala liegen zwischen 1 (überhaupt nicht bzw. fast nie) und 5 (voll und ganz bzw. oft; ebd. S. 101). Die Studie betont, dass die Einleitung zur Abfrage der Items sowie einige der Formulierungen je nach Zielsetzung der Fragestellung mit einem spezifischen Fokus versehen werden könnten (ebd.). Auf Basis eingangs vorgestellter qualitativer Ergebnisse knüpfen wir - wenn auch teils mit deutlicher Formulierungsanpassung in unserer quantitativen Erhebung an die Dimensionen des GEO wie folgt an (vgl. im Detail die Abb. 3 im Anhang):

- Da bei Crowdwork keinerlei Vorgesetztenkontakt besteht, entfällt die Dimension der interpersonalen Gerechtigkeit in diesem Kontext.

- Die prozedurale Gerechtigkeit fokussiert auf das Vorgehen der Entscheidungsfindung (in unserem Fall in Bezug auf Aufgabenstellung und Bewertung) und inwieweit dieser Prozess als beeinflussbar gesehen wird. Drei prozedurale Items legen die Basis für vier Items in der Crowd-Dimension Leistungsbewertung, ein weiteres inspiriert die Formulierung eines Items in Crowd-Dimension Aufgabenbeschreibung.

- Die informationale Gerechtigkeit bezieht sich bei Maier et al. (2007a) auf das Informationsverhalten der entscheidungstreffenden Personen (etwa ob diese wahrheitsgemäß sind oder zeitnah erfolgen), wird in unserem Kontext aber auf die plattformvermittelten Prozesse bezogen. Zwei werden für die Crowd-Dimensionen Leistungsbewertung und Aufgabenbeschreibung verwendet.

- Die distributive Gerechtigkeit bezieht sich auf die Verteilung. Dabei geht es vor allem um die Relation zwischen dem eigenen Beitrag (Qualität und Quantität der geleisteten Arbeit) und dem damit erzielten Ertrag als gerecht betrachtet wird. Zwei distributive GEO-Items stehen Pate für die beiden Items in der Crowd-Dimension Prämien.

Insgesamt stellen wir nachfolgend die Ergebnisse zu neun Items vor, die sich stark auf die organisationalen Gerechtigkeits-Items beziehen, ergänzt um das Crowdspezifische Item zur Planungssicherheit. 
Für unsere quantitative Online-Erhebung wurden Crowdarbeitende auf den deutschsprachigen Onlineplattformen Applause (Testing), Atizo (Innovation) und Crowdguru (Microjobs) über die Plattformen angesprochen, die Befragung selbst wurde über Unipark realisiert. Insgesamt antworteten 230 Crowdarbeitende (Applause: $N=114$, Atizo $N=16$ und Crowdguru $N=100){ }^{2}$

In etwa gleich viele der Beteiligten gaben weibliches und männliches Geschlecht an (jeweils 109, 121), das Median-Alter lag bei 35. Über der Hälfte der Befragten berichten einen tertiären Bildungsabschluss und ähnlich viele geben an, regulär beschäftigt zu sein (bei unklarer Frageformulierung). Die Stichprobe stellt sich damit nicht wesentlich anders dar als andere Studien zu Crowdwork: So sind nach der Studie von Huws et al. (2017) in Deutschland $61 \%$ der Crowdarbeitenden männlich und $39 \%$ weiblich, 52\% sind zwischen 16 und 35 Jahre alt und $63 \%$ arbeiten in einem Vollzeit-Arbeitsverhältnis. Auch nach Pongratz und Bormann sind Crowdarbeitende überwiegend akademisch qualifiziert und in der Mehrheit unter 30 Jahre alt, je nach Studie schwanke der Anteil von Männern zwischen $50 \%$ und $68 \%$ (2017, S. 168). ${ }^{3}$

Insgesamt wurde eine Batterie von 28 Einstellungs-Items mittels einer 5-stufigen Likert-Skala erhoben. Dabei ging es sowohl um Ansprüche an Crowd- wie an normale Erwerbsarbeit. Die meisten Befragten stimmen den meisten Items ,ziemlich“ oder „stark“ zu. Diese geringe Varianz verhindert eine zuverlässige Diagnose von möglichen Mustern in den Antworten. Da die Fragen nicht randomisiert wurden, können mögliche Reihenfolgenartefakte sowie die geänderten Formulierungen die Interpretation etwaiger Kovarianzen erschweren. Schließende Vergleiche zwischen Fragen, Gruppen oder Bedingungen sind aber aus verschiedenen methodischen Gründen ausgeschlossen. Auch eine quantitative Validierung der Dimensionen etwa im Sinne einer konfirmatorischen Faktoranalyse ist mit den vorliegenden Daten nicht möglich. Dem explorativen Charakter dieser Studie folgend stellen wir daher nachfolgend rein kursorisch deskriptive Zählungen vor (auf Atizo wird dabei im Einzelnen wegen der geringen Stichprobengröße nicht eingegangen).

Im Einzelnen stellen wir hier die Ergebnisse zu den oben eingeführten Items für die Dimensionen Planungssicherheit, Leistungsbewertung, Aufgabenbeschreibung und Prämienhöhe vor. Abb. 1 zeigt die Erwartungen an Crowdwork, getrennt nach den drei Plattformen, Abb. 2 stellt die Ansprüche an reguläre Beschäftigung denen an Crowdwork gegenüber.

Die qualitativen Erhebungen illustrierten bereits, dass die fehlende Planungssicherheit für Crowdwork eine spezifische Quelle empfundener Ungerechtigkeit darstellt. Unter anderem deshalb kommt für die meisten Befragten Crowdwork nur als Nebenerwerb in Frage. Eine sichere Auftrags- und Ausschreibungslage (,,si-

\footnotetext{
${ }^{2} \mathrm{Zu}$ weiteren Ergebnissen sowie den Skripten zur Reproduktion der Ergebnisse aus den Rohdaten siehe http://datascience.phil.fau.de/crowd/

${ }^{3}$ Damit unterscheiden sich - trotz vieler anderer Parallelen - Crowdarbeitende von Minijobenden in Deutschland, die weiblicher, verschwindend gering akademisch qualifiziert und gleichmäßiger altersverteilt sind (Zahlen der BA nach Beckmann 2019, S. 170; bei 26\% ist die Ausbildung nicht bekannt). Mit Solo-Selbständigen - einer zweiten nahe liegenden Vergleichsgruppe - lassen sich Crowdarbeitende zwar bezüglich des hohen Anteils von akademischen Abschlüssen vergleichen, hier ist der Anteil von Männern aber etwas höher und vor allem sind sie erheblich älter, erstaunliche $73 \%$ sind 65 Jahre alt oder älter (Brenke und Beznoska 2016, S. 20-25).
} 


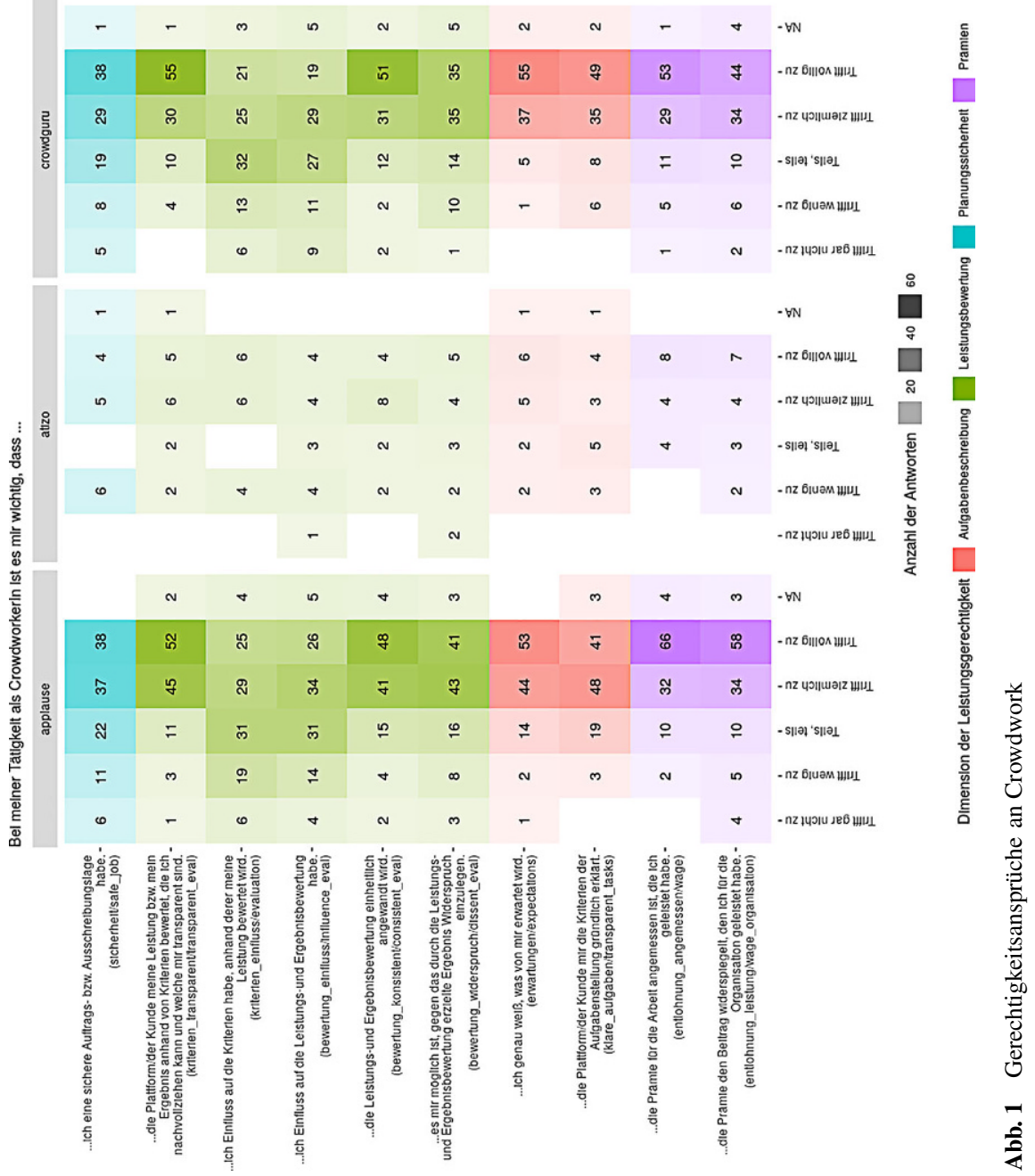

cherheit") wird von den Befragten von Applause und Crowdguru ähnlich wichtig eingeschätzt (Abb. 1, blau).

Die Leistungsbewertung und insbesondere die Möglichkeiten auf diese und ihre Kriterien Einfluss zu nehmen oder auch Widerspruch aussprechen zu können, sind auf Crowd-Plattformen aufgrund der strukturellen Anonymität und Intransparenz ein grundsätzliches Problem, das in den qualitativen Interviews auch entsprechend vieldimensional als erlebte Ungerechtigkeitsdimension geschildert wurde. Die quantitativen Daten zu den Items ,,bewertung_widerspruch“, ,bewertung_konsistent“, ,bewertung_einfluss“, „kriterien_einfluss“ und „kriterien_transparent“ bestätigen die Bedeutung des Themas, allerdings werden die Einflussmöglichkeiten auf die Leistungsbewertung im Vergleich zu anderen Aspekten der Leistungsgerechtigkeit hier scheinbar als relativ weniger wichtig eingeschätzt (Abb. 1, grün). 


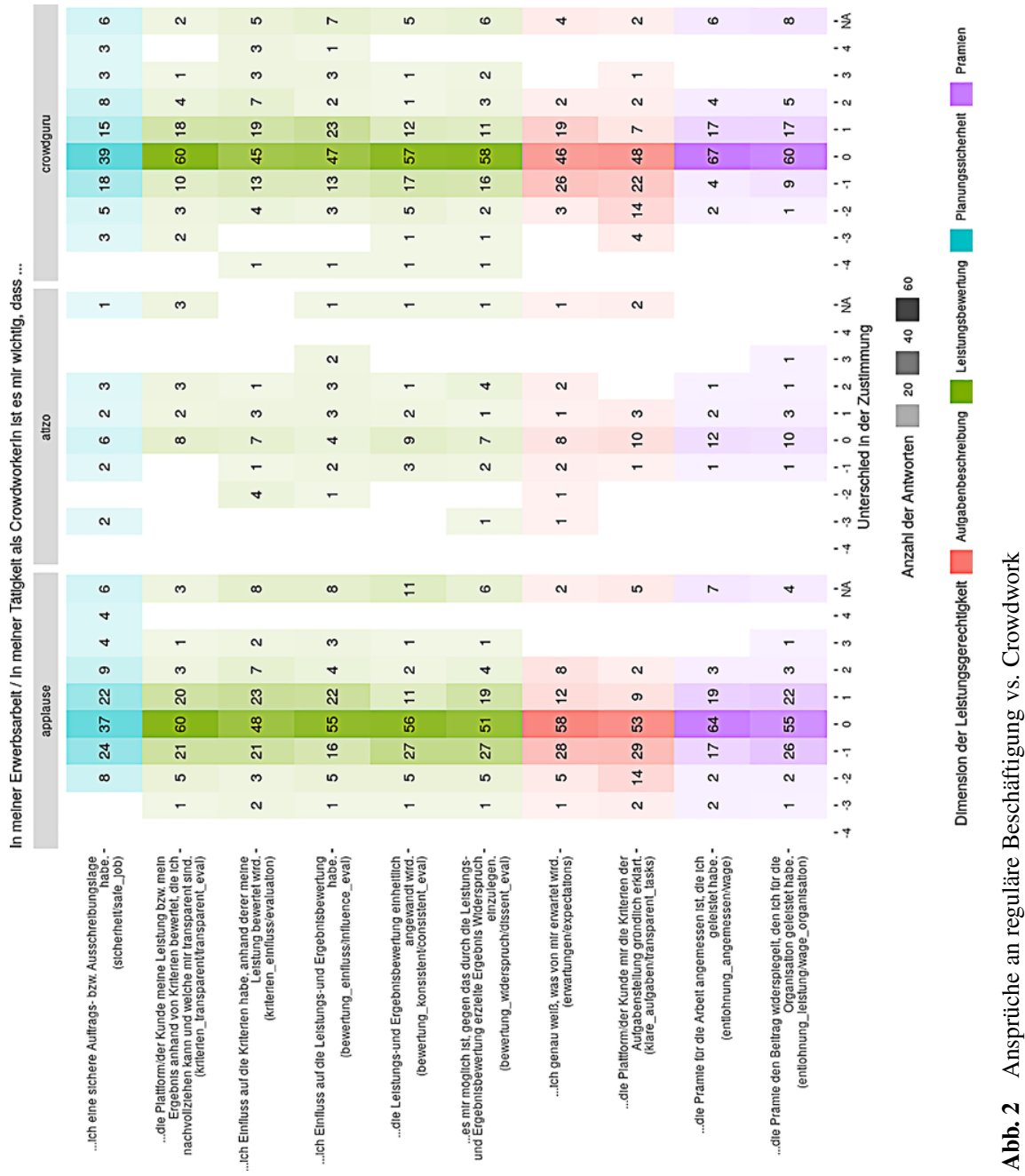

In unklaren oder widersprüchlichen Aufgabenbeschreibungen liegt, wie oben in den qualitativen Ergebnissen geschildert, sehr häufig die Ursache für weitere Ungerechtigkeitserfahrungen. Auch dies scheint sich in den quantitativen Daten zu bestätigen. Die Items „klare_aufgaben“ und ,erwartungen“ werden als wichtig eingeschätzt, möglicherweise sogar wichtiger als in regulärer Beschäftigung (Abb. 2, rot). Ebenfalls konsistent mit den qualitativen Ergebnissen zeigen sich die Zustimmungswerte zu Bedeutung von Prämien für die Items „entlohnung_leistung“ und „entlohnung_angemessen“. (Abb. 1, lila). Allerdings scheint eine angemessene Bezahlung den Befragten in klassischer Beschäftigung ähnlich wichtig zu sein (Abb. 2). 


\section{Same same but different?}

Kronauer (2017) weist - ebenfalls in Bezug auf Dubet - darauf hin, dass innerhalb von abhängiger Erwerbsarbeit als der gegenwärtig vorherrschenden gesellschaftlichen Form von Arbeit, Exklusion und Ungerechtigkeit systematisch angelegt seien, Gerechtigkeit also immer Fiktion bleibe. Dabei sei die Verbindung der Begriffe „Arbeit“ und „Gerechtigkeit“" Folge der Entwicklung der bürgerlich-kapitalistischen Gesellschaft und damit in der Menschheitsgeschichte von eher jungem Datum. Die eigentliche Ungerechtigkeit bestehe darin, dass schon die Inklusion in das Modell Erwerbsarbeit fiktiv mit eigener Leistung, faktisch aber mit vom Individuum nicht beeinflussbaren, übergeordneten Marktmechanismen zusammenhänge (2017, S. 237).

Die hier vorgestellten explorativen Ergebnisse scheinen nahe zu legen, dass sich altbekannte Ansprüche an Gerechtigkeit auch in neuen, digitalen Formen der ent-betrieblichten Arbeit zeigen, auch wenn sie sich wegen des fehlenden Betriebskontexts teils anders ausprägen. Die hier vorgestellten qualitativen und quantitativen Ergebnisse haben trotz ihrer weitgehenden Übereinstimmung explorativen Charakter. Das hat zum einen methodische Gründe: Nicht nur die Institutionen des Arbeitsmarkts, auch die Arbeitsmarktstatistik hat sich an den historischen Formen von Erwerbsarbeit ausgerichtet. Neue Arbeitsformen wie Crowdwork valide zu erfassen, gleicht deshalb einem ,nailing jelly“ (Huws et al. 2017, S. 13); zur Schwierigkeit der Erfassung von Crowdwork und den verschiedenen Erhebungswegen vgl. auch die forschungsmethodischen Reflexionen von Pongratz und Bormann (2017, S. 179-181). Zudem hat auch unsere Studie (wie alle anderen zu Crowdwork), das Problem, dass die Plattformen ihre Datenbasis nicht offenlegen und daher eine valide Einschätzung zur Grundgesamtheit der Crowdarbeitenden systematisch verwehrt bleibt. Der explorative Charakter dieser Studie verbietet es, aus den Ergebnissen Handungsempfehlungen abzuleiten, weder für die betriebliche Praxis von Crowdarbeit nutzenden Unternehmen noch für einen gesetzlichen Regelungsbedarf. Andiskutieren lässt sich auf dieser Basis höchstens: Erstens werden Crowdarbeit einsetzende Unternehmen nicht umhin können - wollen sie qualitativ hochwertige Crowdarbeit dauerhaft als Teil ihrer Wertschöpfungsstrategie einsetzen - sich Plattformen auszusuchen, die offenlegen, ob und wie sie Leistungsrechtigkeit gewährleisten und evaluieren. Zweitens ist davon auszugehen, dass Asprüche an Leistungsgerechtigkeit bei einer Ausweitung von Crowdarbeit zunehmend auch zu einem entsprechenden Regelungsbedarf führen und dass dieser - weil die betriebliche Arena fehlt - plattformübergreifenden Charakter haben dürfte.

Vergleicht man die hier vorgestellten Ergebnisse mit den aktuellen Befunden in der sozialwissenschaftlichen Literatur, so ergibt in Bezug auf den Anspruch auf Leistungsgerechtigkeit eine Gemeinsamkeit: Das Verständnis der Crowdarbeitenden bezieht sich auf einen Begriff von Leistungsgerechtigkeit, welcher überwiegend aufwandsbezogen gedeutet wird und viele Parallelen zu anderen Ansprüchen von Beschäftigten aufweist. Wir wissen allerdings nicht und unsere Datenbasis lässt solche Schlüsse nicht zu, ob es sich dabei um ein Übertragungsphänomen handelt (weil die meisten Befragten im Kern ihren Lebensunterhalt in klassischen Beschäftigungsverhältnissen generieren und die dort entstehenden Gerechtigkeitsansprüche in die 
Online-Welt übertragen) und/oder um ein Übergangsphänomen (das sich historisch in Zukunft dann überleben könnte, wenn klassische Beschäftigtenverhältnisse zu Gunsten von Solo-Selbständigkeit verschwänden). Leistungsgerechtigkeit als Gerechtigkeitsprinzip, so lässt sich trotzdem vorsichtig festhalten, wird im Falle von Crowdwork in Form von Kritik am bestehenden ergebnis- und konkurrenzbasierten System formuliert. 


\section{Anhang}

\section{Tabelle zu Dimensionen der Leistungsgerechtigkeit}

\begin{tabular}{|c|c|c|c|}
\hline $\begin{array}{r}\text { Dimensionen der } \\
\text { Leistungsbewertung }\end{array}$ & Itemkennung & $\begin{array}{l}\text { Bei meiner Tätigkeit ist es mir } \\
\text { wichtig, dass... }\end{array}$ & Maier u.a. 2007 \\
\hline Planungssicherheit & „sicherheit“" & $\begin{array}{l}\text {...ich eine sichere Auftrags- bzw. } \\
\text { Ausschreibungslage habe. }\end{array}$ & - \\
\hline \multirow{5}{*}{ Leistungsbewertung } & $\begin{array}{l}\text { „kriterien } \\
\text { transparent" }\end{array}$ & $\begin{array}{l}\text {... die Plattform/der Kunde meine } \\
\text { Leistung bzw. mein Ergebnis } \\
\text { anhand von Kriterien bewertet, die } \\
\text { ich nachvollziehen kann und } \\
\text { welche mir transparent sind. }\end{array}$ & $\begin{array}{l}\text { 18) Wie sehr waren (seine/ihre) } \\
\text { Erklärungen zum Verfahren } \\
\text { nachvollziehbar? } \\
\text { (Informational; 0,803) }\end{array}$ \\
\hline & $\begin{array}{l}\text { "kriterien } \\
\text { einfluss“- }\end{array}$ & $\begin{array}{l}\text {...dass ich Einfluss auf die } \\
\text { Kriterien habe, anhand derer } \\
\text { meine Leistung bewertet wird. }\end{array}$ & \multirow{2}{*}{$\begin{array}{l}\text { 02) Ich möchte auf Ergebnisse, die } \\
\text { mich betreffen, Einfluss nehmen } \\
\text { können. } \\
\text { (Prozedural; 0,613) }\end{array}$} \\
\hline & $\begin{array}{l}\text { „bewertung } \\
\text { einfluss“ }\end{array}$ & $\begin{array}{l}\text {...ich Einfluss auf die Leistungs- } \\
\text { und Ergebnisbewertung habe. }\end{array}$ & \\
\hline & $\begin{array}{l}\text { „bewertung } \\
\text { konsistent“- }\end{array}$ & $\begin{array}{l}\text {...die Leistungs-und } \\
\text { Ergebnisbewertung einheitlich } \\
\text { angewandt wird. }\end{array}$ & $\begin{array}{l}\text { 03) Wie sehr wurde das } \\
\text { (Vorgehen) einheitlich angewandt? } \\
\text { (Prozedural; } 0,519 \text { ) }\end{array}$ \\
\hline & $\begin{array}{l}\text { „bewertung } \\
\text { widerspruch“ }\end{array}$ & $\begin{array}{l}\text {...es mir möglich ist, gegen das } \\
\text { durch die Leistungs- und } \\
\text { Ergebnisbewertung erzielte } \\
\text { Ergebnis Widerspruch einzulegen. }\end{array}$ & $\begin{array}{l}\text { 06) Wie sehr war es Ihnen } \\
\text { möglich, gegen das durch das } \\
\text { (Vorgehen) erzielte Ergebnis } \\
\text { Widerspruch einzulegen? } \\
\text { (Prozedural; 0,600) }\end{array}$ \\
\hline \multirow[b]{2}{*}{ Aufgabenbeschreibung } & „erwartungen“" & $\begin{array}{l}\text {..ich genau weiß, was von mir } \\
\text { erwartet wird. }\end{array}$ & $\begin{array}{l}\text { 05) Wie sehr basierte das } \\
\text { (Vorgehen) auf zutreffenden } \\
\text { Informationen? } \\
\text { (Prozedural; 0,702) }\end{array}$ \\
\hline & $\begin{array}{l}\text { „klare } \\
\text { aufgaben“ }\end{array}$ & $\begin{array}{l}\text {...die Plattform/der Kunde mir die } \\
\text { Kriterien der Aufgabenstellung } \\
\text { gründlich erklärt. }\end{array}$ & $\begin{array}{l}\text { 17) Wie sehr hat (der/die } \\
\text { Vorgesetzte) das Verfahren } \\
\text { gründlich erklärt? } \\
\text { (Informational; } 0,780)\end{array}$ \\
\hline \multirow[b]{2}{*}{ Prämien } & $\begin{array}{l}\text {,entlohung__ } \\
\text { angemessen“ }\end{array}$ & $\begin{array}{l}\text {... die Prämie für die Arbeit } \\
\text { angemessen ist, die ich geleistet } \\
\text { habe. }\end{array}$ & $\begin{array}{l}\text { 09) Wie sehr ist Ihr (Ergebnis) } \\
\text { angemessen für die Arbeit, die Sie } \\
\text { geleistet haben? } \\
\text { (Distributiv, 0,948) }\end{array}$ \\
\hline & $\begin{array}{l}\text { „entlohnung } \\
\text { leistung“ }\end{array}$ & $\begin{array}{l}\text {...die Prämie den Beitrag } \\
\text { widerspiegelt, den ich für die } \\
\text { Organisation geleistet habe. }\end{array}$ & $\begin{array}{l}\text { 10) Wie sehr spiegelt Ihr } \\
\text { (Ergebnis) den Beitrag wider, } \\
\text { den Sie für die Organisation } \\
\text { geleistet haben? } \\
\text { (Distributiv, } 0,856 \text { ) }\end{array}$ \\
\hline
\end{tabular}

Abb. 3 Übersicht verwendeter Items zur Leistungsgerechtigkeit 
Open Access Dieser Artikel wird unter der Creative Commons Namensnennung 4.0 International Lizenz (http://creativecommons.org/licenses/by/4.0/deed.de) veröffentlicht, welche die Nutzung, Vervielfältigung, Bearbeitung, Verbreitung und Wiedergabe in jeglichem Medium und Format erlaubt, sofern Sie den/die ursprünglichen Autor(en) und die Quelle ordnungsgemäß nennen, einen Link zur Creative Commons Lizenz beifügen und angeben, ob Änderungen vorgenommen wurden.

\section{Literatur}

Alpar P, Osterbrink L (2018) Antecedents of perceived fairness in pay for microtask crowdwork. ECIS 2018 Proceedings at AIS Electronic Library

Beckmann F (2019) Minijobs in Deutschland. Die subjektive Wahrnehmung von Erwerbsarbeit in geringfügigen Beschäftigungsverhältnissen. Springer, Wiesbaden

Benner C (Hrsg) (2014) Crowdwork - Zurück in die Zukunft? Perspektiven digitaler Arbeit. Bund-Verlag, Köln

Berg J (2016) Income security in the on-demand ecomomy: findings and policy lessons from a survey of crowdworkers. Comp Labor Law Policy J 37:0-26

BMAS (2015) Arbeit weiter denken. Grünbuch Arbeiten 4.0. Bundesministerium für Arbeit und Soziales, Berlin

Bollier D (2011) The future of work. What it means for individuals, businesses, markets and governments. The Aspen Institute, Washington D.C.

Breisig T (2018) Arbeiten Sie noch oder leisten Sie schon? Ein Essay über Entwicklungen und Praktiken der betrieblichen Leistungspolitik. Z Arbeitswiss 72:185-189

Brenke K, Beznoska M (2016) Solo-Selbständige in Deutschland - Strukturen und Erwerbsverläufe. DIW, Berlin

Colquitt JA (2001) On the dimensionality of organizational justice: a construct validation of a measure. J Appl Psychol 86:386-400. https://doi.org/10.1037/0021-9010.86.3.386

Dabrowski M, Wolf J (Hrsg) (2017) Crowdworking und Gerechtigkeit auf dem Arbeitsmarkt. Ferdinand Schöningh, Münster

Deutschen Crowdsourcing Verband (2017) Code of Conduct. Grundsätze für bezahltes Crowdsourcing/ Crowdworking. http://www.crowdsourcing-code.de/. Zugegriffen: 20. März 2019

Dubet F (2008) Ungerechtigkeiten: Zum subjektiven Ungerechtigkeitsempfinden am Arbeitsplatz. Hamburger Edition, Hamburg

Glaser BG, Strauss AL (2009) The discovery of grounded theory: strategies for qualistative research. Aldine, New Brunswick

Gläser J, Laudel G (2010) Experteninterviews und qualitative Inhaltsanalyse als Instrumente rekonstruierender Untersuchungen. VS, Wiesbaden

Graham M, Hjorth I, Lehdonvirta V (2017) Digital labour and development: impacts of global digital labour platforms and the gig economy on worker livelihoods. Transfer 23:135-162. https://doi.org/ $10.1177 / 1024258916687250$

Hertwig M, Papsdorf C (2017) Varieties of Sharing. Handlungsorientierungen, Strukturen und Arbeitsbedingungen eines neuartigen Feldes. Berl J Soziol 27:3-4. https://doi.org/10.1007/s11609-018-03595

Howcroft D, Bergvall-Kåreborn B (2019) A typology of crowdwork platforms. Work Employ Soc 33:21-38. https://doi.org/10.1177/0950017018760136

Hürtgen S, Voswinkel S (2014) Nichtnormale Normalität? Anspruchslogiken aus der Arbeitnehmermitte. Edition Sigma, Berlin

Huws U, Spencer NH, Syrdal DS, Holts K (2017) Work in european gig-economy: research results from the UK, Sweden, Germany, Austria, the Netherlands, Switzerland and Italy. http://www.uni-europa.org/ wp-content/uploads/2017/11/europeagigeconomy-longversionpdf.pdf. Zugegriffen: 26. Juni 2019

Kagermann H, Lukas W-D, Wahlster W (2011) Industrie 4.0: Mit dem Internet der Dinge auf dem Weg zur 4. industriellen Revolution. http://www.vdi-nachrichten.com/Technik-Gesellschaft/Industrie-40Mit-Internet-Dinge-Weg-4-industriellen-Revolution. Zugegriffen: 20. März 2019

Kawalec S (2019) Arbeit in der Cloud. Universität Hohenheim, Hohenheim (Dissertation)

Kratzer N, Menz W, Tullius K, Wolf H (2015) Legitimationsprobleme in der Erwerbsarbeit: Gerechtigkeitsansprüche und Handlungsorientierungen in Arbeit und Betrieb. Edition Sigma, Baden-Baden

Kronauer M (2017) Arbeit, Exklusion und Ungerechtigkeit. In: Misselhorn C, Behrendt H (Hrsg) Arbeit, Gerechtigkeit und Inklusion. Wege zu gleichberechtigter gesellschaftlicher Teilhabe. J.B. Metzler, Stuttgart, S 229-238 
Maier GW, Streicher B, Jonas E, Frey D (2007a) Bedürfnisse nach organisationaler Gerechtigkeit und Bereitschaft zu innovativem Handeln. Wirtschaftspsychologie 9:43-54

Maier GW, Streicher B, Jonas E, Woschée R (2007b) Gerechtigkeitseinschätzungen in Organisationen. Die Validität einer deutschsprachigen Fassung des Fragebogens von Colquitt (2001). Diagnostica 53:97-108

Mandl I, Curtarelli M (2017) Crowd employment and ICT-based mobile work-new employment forms in europe. In: Meil P, Kirov V (Hrsg) Policy implications of virtual work. Palgrave Macmillan, Cham, S $51-79$

Menz W, Nies S (2018) Doing Inequality at Work. Zur Herstellung und Bewertung von Ungleichheiten in Arbeit und Betrieb. In: Behrmann L, Eckert F, Gefken A, Berger PA (Hrsg) Doing Inequality‘. Prozesse sozialer Ungleichheit im Blick qualitativer Sozialforschung. Springer, Wiesbaden, S 123-147

Menz W, Tomazic AC (2017) Gerechte neue Arbeitswelt? Crowdworking aus normativ-empirischer Perspektive der Soziologie. In: Dabrowski M, Wolf J (Hrsg) Crowdworking und Gerechtigkeit auf dem Arbeitsmarkt. Ferdinand Schöningh, Paderborn, S 9-32

Mrass V, Leimeister JM (2018) Crowdworking-Plattformen als Enabler neuer Formen der Arbeitsorganisation. In: Fortmann HR, Kolocek B (Hrsg) Arbeitswelt der Zukunft. Springer, Wiesbaden, S 139-152

Papsdorf C (2009) Wie Surfen zu Arbeit wird: Crowdsourcing im Web 2.0. Campus, Frankfurt am Main

Petriglier G, Ashford SJ, Wrzesniewski A (2018) Agony and ecstasy in the gig economy: cultivating holding environments for precarious and personalized work identities. Adm Sci Q 64(1):124-170

Pongratz HJ, Bormann S (2017) Online-Arbeit auf Internet-Plattformen. Empirische Befunde zum Crowdworking in Deutschland. Arbeits-und Industriesoziologische Studien AIS 10, S 158-181

Przyborski A, Wohlrab-Sahr M (2010) Qualitative Sozialforschung: Ein Arbeitsbuch. Oldenbourg, München

Schneider H (2018) Wahrgenommene Lohngerechtigkeit in Deutschland. Leistung, Bedarf, Chancengerechtigkeit - Worauf basieren gerechte Löhne? List Forum 44:357-378. https://doi.org/10.1007/ s41025-018-0101-2

Tullius K, Wolf H (2016) Moderne Arbeitsmoral: Gerechtigkeits- und Rationalitätsansprüche von Erwerbstätigen heute. WSI Mitt 68:493-502

Ye T, You S, Robert LP Jr. (2017) When does more money work? Examining the role of perceived fairness in pay on the performance quality of crowdworkers. In: Proceedings of the eleventh international AAAI conference on web and social media (ICWSM 2017). AAAI Press, Palo Alto, S 327-336 